\title{
CONTINUUM FLUX AND POLARIZATION IN B[e]
}

\section{SUPERGIANTS}

\author{
F.X. DE ARAÚJO, PH. STEE and J. LEFÈVRE \\ Observatoire de la Côte D'Azur, B.P. 229 Nice Cedex 4, 06304 - France
}

$\mathrm{B}[\mathrm{e}]$ supergiants constitute one group of stars in the upper left part of the HR diagram. On the basis of their hybrid spectrum and their intrinsic polarization a two components envelope was suggested (Zickgraf \& SchulteLadbeck, 1989): a "polar wind" and a denser "equatorial disc".

Recently Araújo et al (1993) have presented an axi-symmetric model in which the radiative line force varies from pole to equator. Some typical density profiles (arising from the application to $\mathrm{B}[\mathrm{e}]$ supergiants) may be seen in fig. 1. In this particular model it was adopted $M=30 M_{\odot}, R=75 R_{\odot}$, $T_{\text {eff }}=20000^{\circ} \mathrm{K}$ and a rotational rate of $70 \%$ of the critical speed. There is a density enhancement towards the equatorial plane which is due to a greater mass flux and a lower expansion.

Assuming a fully ionized, pure hydrogen envelope the density laws give directly the electronic distribution. (A numerical code is being developed in order to overcome this drastic hypothesis, see Stee \& Araújo, this Symposium). Fig. 2 shows the optical thickness due to electronic absorption as a function of wavelength (lines) and that due to electronic scattering (circles), which is $\lambda$ independent. It is clearly seen as opacity increases from pole to equator.

Subsequently the propagation of stellar radiation in the envelope was followed by employing a Monte Carlo type scheme (Lefevre \& Daniel, 1988). Figure 3 shows the flux that escapes from the envelope as a function of $\lambda$ at some inclination angles: $i=0^{\circ}, i=45^{\circ}$ and $i=90^{\circ}$. We see that $\mathrm{i}$ ) emission decreases with $\lambda$ (the star radiates as a blackbody with $T=2.10^{4} K$ ); ii) the flux is dependent on the line of sight due to different opacities; and iii) the wavelength dependence of absorption is found in the increasing separation of lines. In fig. 4 we plot the global flux that escapes in all directions as a function of the number of scatterings. We may conclude that the single scattering approximation is not reasonable since at least $50 \%$ of radiation is scattered twice or more.

Concerning polarisation degrees we stress they are preliminary ones since we have not been able yet to perform computations with a number of interactions great enough to prevent statistical fluctuations. Fig. 5 shows that we have obtained degrees between about $0.5 \%$ and $4.5 \%$, increasing with inclination angles. On the other hand we can see in fig. 6 that no dependence on wavelength was obtained. This result however must be kept with great caution since we have not included the envelope emission. It will most likely 
add up a no (or weakly) polarised flux which is strongly increasing with $\lambda$. In order to compare the results with observational data we must taking into account ionisation equilibrium, including envelope emission and performing more accurate numerical simulations.

\section{References}

Araújo, F.X., Freitas Pacheco, J.A. \& Petrini, D.: 1993, Monthly Notices of the RAS , in press

Lefévre, J. \& Daniel, J.-Y.: 1988, in "Polarized radiation of circumstellar origin", ( $G$. Coyne et al., eds.), Vatican Observatory, 523

Zickgraf, F.-J., \& Schulte-Ladbeck, R.: 1989, Astronomy and Astrophysics 214, 274

figure 1

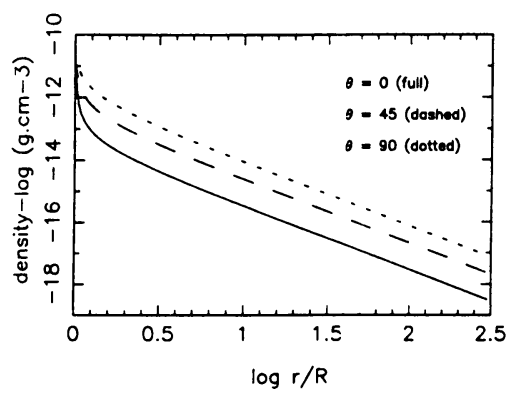

figure 3

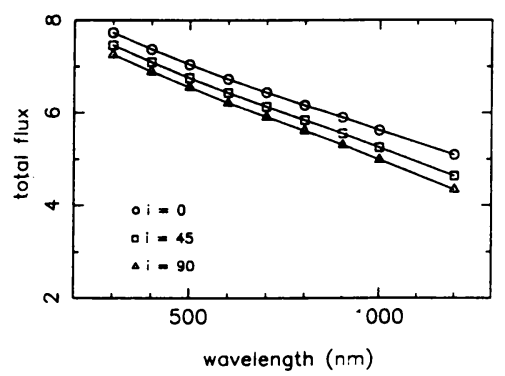

figure 5

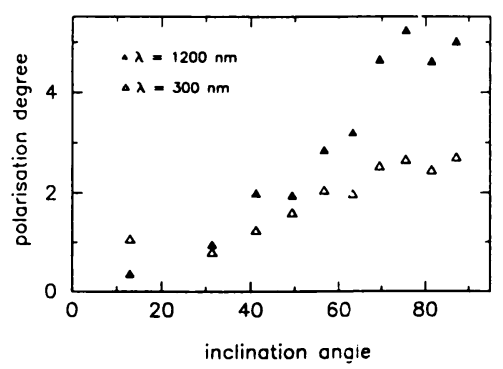

figure 2

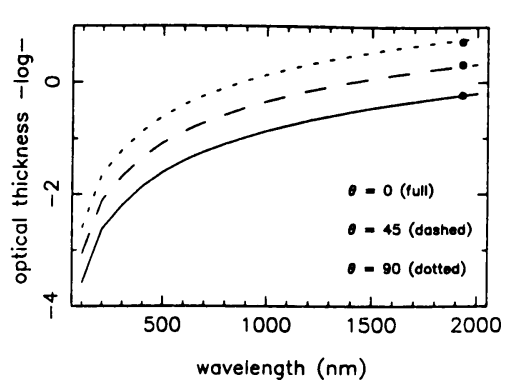

figure 4

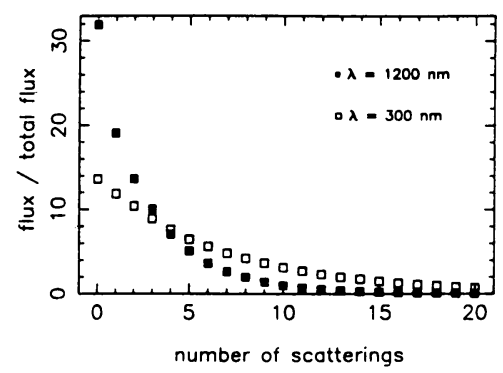

figure 6

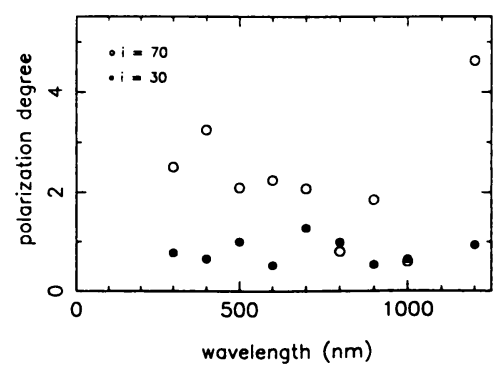

